University of New Orleans

ScholarWorks@UNO

$4-1973$

\title{
Trajectories Describing the Evolution of Polarized Light in Homogeneous Anisotropic Media and Liquid Crystals
}

\author{
Rasheed M.A. Azzam \\ University of New Orleans, razzam@uno.edu
}

N. M. Bashara

B. E. Merrill

Follow this and additional works at: https://scholarworks.uno.edu/ee_facpubs

Part of the Electrical and Electronics Commons

\section{Recommended Citation}

R. M. A. Azzam, B. E. Merrill, and N. M. Bashara, "Trajectories Describing the Evolution of Polarized Light in Homogeneous Anisotropic Media and Liquid Crystals," Appl. Opt. 12, 764-771 (1973) http://www.opticsinfobase.org/ao/abstract.cfm?URI=ao-12-4-764.

This Article is brought to you for free and open access by the Department of Electrical Engineering at ScholarWorks@UNO. It has been accepted for inclusion in Electrical Engineering Faculty Publications by an authorized administrator of ScholarWorks@UNO. For more information, please contact scholarworks@uno.edu. 


\title{
Trajectories Describing the Evolution of Polarized Light in Homogeneous Anisotropic Media and Liquid Crystals
}

\author{
R. M. A. Azzam, B. E. Merrill, and N. M. Bashara
}

\begin{abstract}
Trajectories are given that describe the evolution of the ellipse of polarization in the complex plane for light propagating in a homogeneous anisotropic medium and along the helical axis of a cholesteric liquid crystal. For the general homogeneous anisotropic medium that exhibits combined birefringence and dichroism the trajectory is a spiral that converges to the low-absorption eigenpolarization. For pure birefringence the trajectory becomes a complete circle that encloses one eigenpolarization, whereas for pure dichroism the trajectory becomes an arc of a circle that ends at the low-absorption eigenstate. The case of a cholesteric (or twisted nematic) liquid crystal leads to an interesting family of trajectories that can be considered as distorted hypo- or epicycloids. These trajectories are nonrepetitive (open) and may show multilobes or branches depending upon the initial polarization and the properties of the liquid crystal. Graphs are also presented where the ellipticity and azimuth are plotted separately as functions of distance along the helical axis.
\end{abstract}

\section{Introduction}

Recently we have shown that the evolution of the ellipse of polarization of light passing through an anisotropic medium is governed by Riccati's first-order differential equation ${ }^{1}$

$$
d \chi / d z=n_{12} \chi^{2}+\left(n_{22}-n_{11}\right) \chi+n_{21},
$$

where $\chi$ is a complex variable that determines the ellipse of polarization at a distance $z$ and $n_{i j}$ are the elements of the differential propagation Jones matrix $N$ of the medium. ${ }^{2}$ The solution of Eq. (1) was derived for a homogeneous anisotropic medium and for a cholesteric liquid crystal when light propagates along the helical axis. For the homogeneous anisotropic medium the solution is given by

$$
\begin{aligned}
\chi\left(z, \chi_{0}\right)= & \left\{\left[\beta-\frac{1}{2}\left(n_{11}-n_{22}\right) \tan \beta z\right] \chi_{0}\right. \\
& \left.+\left(n_{21} \tan \beta z\right)\right\} /\left\{\left(n_{12} \tan \beta z\right) \chi_{0}\right. \\
& \left.+\left[\beta+\frac{1}{2}\left(n_{11}-n_{22}\right) \tan \beta z\right]\right\},
\end{aligned}
$$

where

$$
\beta=\left[-\frac{1}{4}\left(n_{11}-n_{22}\right)^{2}-n_{12} n_{21}\right]^{1 / 2}
$$

The authors are with the Electrical Materials Laboratory, Department of Electrical Engineering, University of Nebraska, Lincoln, Nebraska 68508.

Received 14 August 1972. $\chi\left(z, \chi_{0}\right)$ describes the ellipse of polarization after light has propagated through a distance $z$ starting from an initial polarization state $\chi_{0}$ at $z=0$. Both $\chi$ and $N$ are defined using the orthogonal $x$ and $y$ linear polarization as basis states.

For the cholesteric liquid crystal the solution is

$$
\bar{\chi}\left(z, \chi_{0}\right)=\frac{\left.[\beta-i \alpha \tan \beta z) \chi_{0}+\left(-i g_{0} \tan \beta z\right)\right]}{\left[\left(-i g_{0} \tan \beta z\right) \chi_{0}+(\beta+i \alpha \tan \beta z)\right] \exp (i 2 \alpha z)},
$$

where

$$
\begin{gathered}
\alpha=2 \pi / p, \\
\beta=g_{0} /\left[\left(\alpha / g_{0}\right)^{2}+1\right]^{1 / 2} .
\end{gathered}
$$

$p$ is the pitch of the helical structure and $g_{0}$ is a measure of the birefringence of the molecular planes. ${ }^{3}$ In Eq. (4) $\chi$ is defined using the orthogonal left and right circular polarizations as basis states. Obviously the results for cholesteric liquid crystals also apply to the twisted nematics.

In Ref. 1 we have examined the trajectory of $\chi\left(z, \chi_{0}\right)$ only for special cases of Eqs. (2) and (4). In the present paper we examine the general nature of these analytic solutions.

\section{Homogeneous Anisotropic Media}

To examine the nature of the trajectory described by Eq. (2) for any (constant) $N$ matrix we substitute for $\tan \beta z$ in that equation the expression 


$$
\tan \beta z=i[1-\exp (i 2 \beta z)] /[1+\exp (i 2 \beta z)],
$$

which gives

$$
\chi\left(z, \chi_{0}\right)=[A+B \exp (i 2 \beta z)] /[C+D \exp (i 2 \beta z)] \text {, }
$$

where

$$
\begin{aligned}
& A=i n_{21}+\left[\beta-\frac{1}{2} i\left(n_{11}-n_{22}\right)\right] \chi_{0} \\
& B=-i n_{21}+\left[\beta+\frac{1}{2} i\left(n_{11}-n_{22}\right)\right] \chi_{0} \\
& C=\left[\beta+\frac{1}{2} i\left(n_{11}-n_{22}\right)\right]+i n_{12} \chi_{0} \\
& D=\left\{\left[\beta-\frac{1}{2} i\left(n_{11}-n_{22}\right)\right]-i n_{12} \chi_{0} .\right.
\end{aligned}
$$

Equation (7) is interesting because it shows that the evolution of $\chi\left(z, \chi_{0}\right)$ with $z$, starting from an initial polarization $\chi_{0}$ at $z=0$, is an image through a bilinear transformation of the evolution of $\exp (\mathrm{i} 2 \beta z)$ with $z$. The coefficients $A, B, C$, and $D$ of this bilinear transformation are linear functions of the initial polarization state $\chi_{0}$ as evident from Eq. (8). It can be proved ${ }^{4}$ that the ratios $A / C$ and $B / D$ are independent of $\chi_{0}$ and give the two eigenpolarizations $\chi_{e 1}$ and $\chi_{e 2}$ of the medium, i.e.,

$$
\chi_{e 1}=A / C, \quad \chi_{e 2}=B / D .
$$

In terms of $\left(n_{i j}\right)$ the eigenpolarizations are given by

$$
\begin{aligned}
\chi_{e 1,2}=\left(1 / 2 n_{12}\right)\left\{\left(n_{22}-\right.\right. & \left.n_{11}\right) \\
& \left. \pm\left[\left(n_{22}-n_{11}\right)^{2}+4 n_{12} n_{21}\right]^{1 / 2}\right\}
\end{aligned}
$$

Equation (10) has the same form as Eq. (16) of Ref. 5 , which gives the eigenpolarizations of an optical system in terms of its Jones matrix.

From the above we have. seen that $\chi\left(z, \chi_{0}\right)$ is obtained from $\exp (i 2 \beta z)$ by a bilinear transformation. Therefore, to determine the trajectory of $\chi\left(z, \chi_{0}\right)$ we consider the behavior of $\exp (i 2 \beta z)$ as $z$ is increased from zero to infinity. This leads to the following three cases.
Case i, $\beta$ Real

In this case $\exp (i 2 \beta z)$ moves uniformly around the unit circle in the complex plane starting from the point $(1,0)$ on the real axis when $z=0$. This motion is counterclockwise or clockwise depending on whether $\beta$ is positive or negative, respectively [Fig. 1(a)]. One revolution around the unit circle is completed each time $z$ is increased a distance $d$ given by

$$
d=\pi / \beta .
$$

Because a bilinear transformation maps a circle into a circle, the locus of $\chi\left(z, \chi_{0}\right)$ is a circle through $\chi_{0}$ that is traversed as $z$ is increased [Fig. 1(b)]. The solution is obviously periodic with a period given by Eq. (11).

\section{Case II, $\beta$ Imaginary}

As $z$ is increased from zero to infinity $\exp (i \dot{2} \beta z)$ moves on the real axis from the point $(1,0)$ to the origin if $\beta$ is positive and to infinity if $\beta$ is negative [Fig. 2(a)]. The real axis is a degenerate circle that is mapped by a bilinear transformation into a circle in the complex plane. Because $\exp (i 2 \beta z)$ scans only one segment of the real axis and does that only once (as $z$ is changed from zero to infinity), the evolution of $\chi\left(z, \chi_{0}\right)$ is aperiodic and proceeds along an arc of a circle [Fig. 2(b)] from the initial state $\chi_{0}$ to a final state $\chi_{f}$ given by

$$
\chi_{f}=A / C=\chi_{e 1}
$$

or

$$
\chi_{f}=B / D=\chi_{e 2}
$$

Equations (12a) and (12b) are obtained by setting $\exp (i 2 \beta z)$ equal to zero and infinity, respectively, in Eq. (7) and directly using Eq. (9). This shows that the ellipse of polarization converges asymptotically (in a nonoscillatory fashion) to one of the two eigenpolarizations, obviously the one of lower absorption.

Based on cases I and II above we are led to the following important conclusion. If the differential propagation Jones matrix $N$ of a homogeneous anisotropic medium is such that $\beta$ [Eq. (3)] is either real or imaginary, the evolution of the ellipse of polariza-

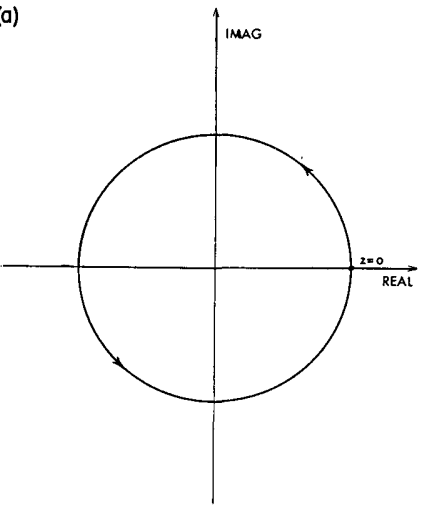

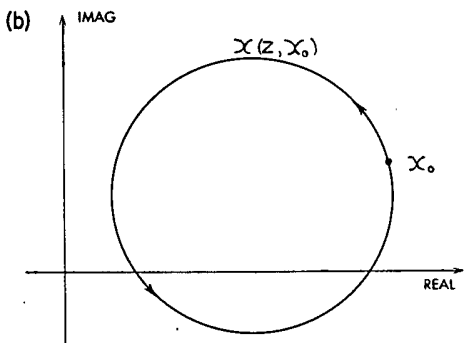

Fig. 1. (a) The function $\exp (i 2 \beta z)$ for $\beta$ real and positive gives the unit circle in the complex planes. The rotational sense is reversed when $\beta$ is negative. (b) The trajectory of $\chi\left(z, \chi_{0}\right)$ is an image through a bilinear transformation [Eq. (7)] of the unit circle of (a). $\chi\left(z, x_{0}\right)$ is a circle that is traversed periodically as $z$ is increased starting from the initial state $\chi_{0}$ at $z=0$. 
(a)

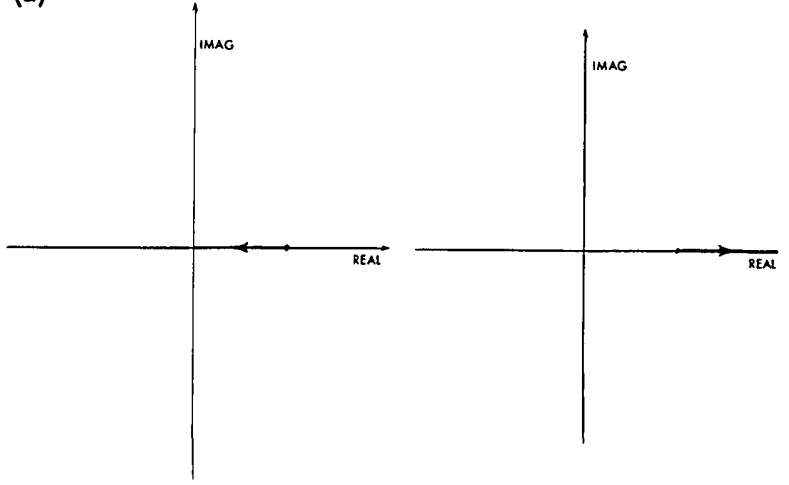

(b)

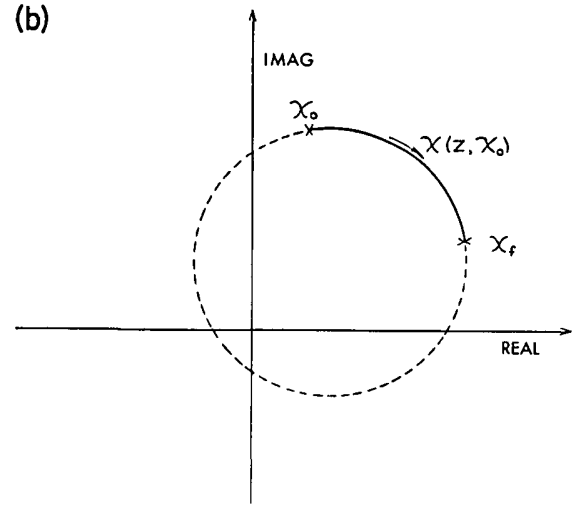

Fig. 2. (a) The function $\exp (i 2 \beta z)$ when $\beta$ is purely imaginary and positive (left) or negative (right). (b) $\chi\left(z, \chi_{0}\right)$, which is the image of $\exp (i 2 \beta z)$ in (a), follows an arc of a circle that starts at initial state $\chi_{0}(z=0)$ and converges asymptotically to the final state $\chi_{f}(z=\infty) \cdot \chi_{f}$ is the low-absorption eigenpolarization.

tion is along a circle in the complex plane and on the Poincare sphere. The last part of this conclusion follows from the circle-preserving property of the stereographic projection that relates the complex-plane and Poincaré-sphere representations of polarized light. ${ }^{6}$ An $N$ matrix that leads to $\beta$ that is either real or imaginary satisfies the condition that

$$
\operatorname{Im}\left[\frac{1}{4}(\operatorname{Tr} N)^{2}-(\operatorname{Det} N)\right]=0,
$$

as can be seen from the expression of $\beta$ in Eq. (3). $\operatorname{Tr} N$ and Det $N$ stand for the trace and determinant of $N$, respectively, and Im signifies the imaginary part of.

Two common types of anisotropy that satisfy Eq. (13) are elliptic birefringence (eb) and elliptic dichroism (ed). In an elliptically birefringent medium there are two orthogonally polarized waves that propagate with different velocities and equal attenuation with their polarization unaffected. In an elliptically dichroic medium the two eigenpolarizations are attenuated at different rates but propagate with the same velocity. The $N$ matrix for these two types of anisotropy takes the form

$$
N_{\mathrm{eb}}=\left[\begin{array}{ll}
A & B \\
-B^{*} & A^{*}
\end{array}\right], \quad N_{\mathrm{ed}}=\left[\begin{array}{ll}
p & R \\
R^{*} & q
\end{array}\right],
$$

where $A, B$, and $R$ are complex and $p$ and $q$ are real. Both $N_{\text {eb }}$ and $N_{\text {ed }}$ have real trace and real determinant and thus satisfy Eq. (13). Furthermore $N_{\mathrm{eb}}$ leads to a real value of $\beta$ corresponding to case I and $N_{\text {ed }}$ leads to an imaginary value for $\beta$ corresponding to case II.

\section{Case III, $\beta$ Complex}

Writing $\beta=\beta_{r}+i \beta_{i}, \exp (i 2 \beta z)$ becomes $\exp \left(-2 \beta_{i} z\right) \exp \left(i 2 \beta_{r} z\right)$. As $z$ varies from zero to infinity $\exp (i 2 \beta z)$ describes a logarithmic spiral [Fig. $3(\mathrm{a})$ ] that starts at $(1,0)$ and converges to the origin if $\beta_{i}$ is positive or blows to infinity if $\beta_{i}$ is negative. The sense of description is counterclockwise or clock-
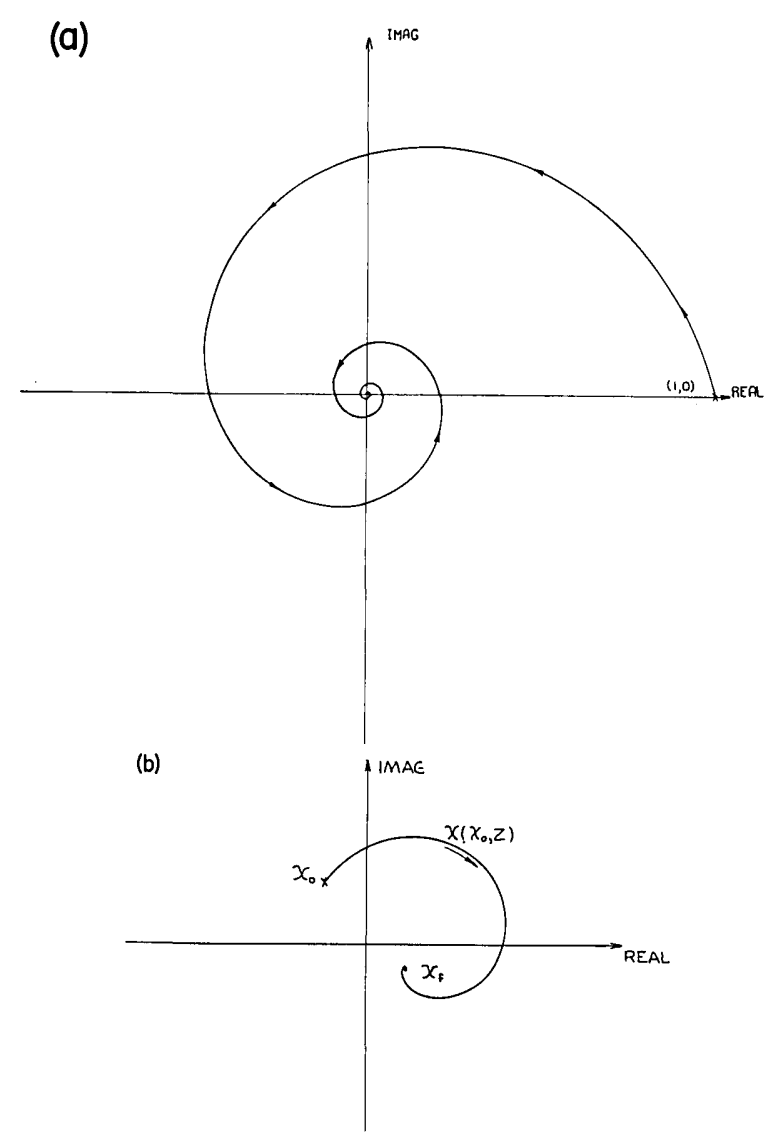

Fig. 3. (a) The function $\exp (i 2 \beta z)$ for $\beta$ complex $\left(\beta=\beta_{r}+i \beta_{i}\right)$ is a logarithmic spiral. Reversing the sign of $\beta_{i}$ causes the spiral to expand out to infinity. The sense of the spiral is clockwise (instead of counterclockwise) when $\beta_{r}$ is negative. (b) $\chi\left(z, \chi_{0}\right)$, being an image of the spiral of (a), is also a spiral. The final state $\chi_{f}$ is the low-absorption eigenpolarization.

wise dependent on whether the sign of $\beta_{r}$ is positive or negative. According to Eq. (7) the image trajectory $\chi\left(z, \chi_{0}\right)$ starts at $\chi_{0}(z=0)$ and approaches, in a spiral fashion, the eigenpolarization $\chi_{e 1}=A / C(z=$ 
(a)

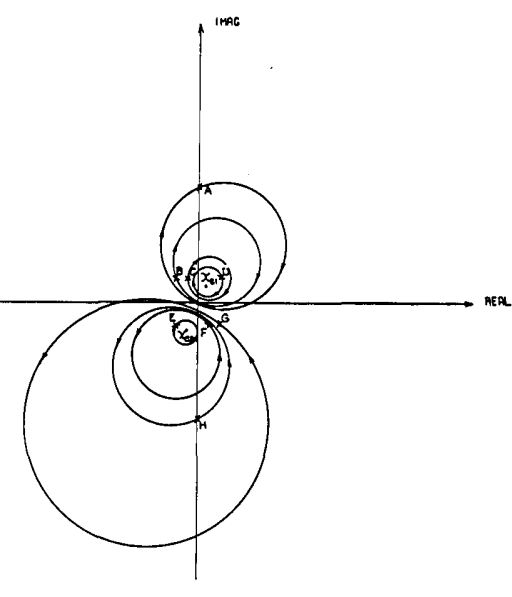

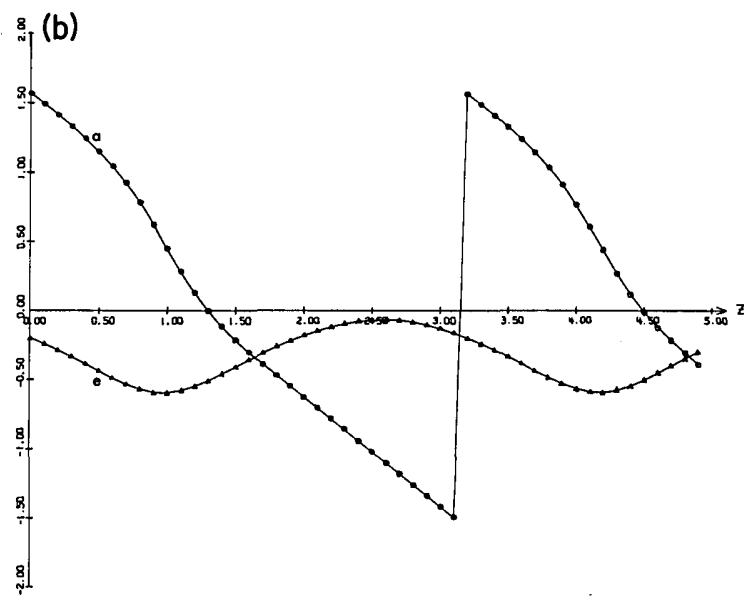

Fig. 4. (a) The computed evolution of the ellipse of polarization $\left[\chi\left(z, \chi_{0}\right)\right]$ in an elliptically birefringent medium with eigenpolarizations $\chi_{e 1}$ and $\chi_{e 2}$ for eight different initial polarization states $A, B ., \ldots$ and $H$. The $N$ matrix of the medium has the form of $N_{\mathrm{eb}}$ in Eq. (14) with $A=0.75+i .25$ and $B=0.875+i .375$. (b) The variation of the ellipticity $e(\Delta)$ and azimuth $a(0)$ with distance $z$ along the direction of propagation in the elliptically birefringent medium corresponding to the trajectory that starts at $H$ in (a). Note that both functions are periodic with $z$.

$\infty, \beta_{i}$ positive $)$ or $\chi_{e 2}=B / D\left(z=\infty, \beta_{i}\right.$ negative $)$. The final state is necessarily the one of lower absorption. That the logarithmic spiral of $\exp (i 2 \beta z)$ leads to a spiral for $\chi\left(z, \chi_{0}\right)$ [Fig. $3(\mathrm{~b})$ ] is a consequence of the conformal nature of the bilinear transformation. ${ }^{7}$ From the relation between the complex plane and the Poincare sphere it also follows that the representative point converges spirally towards the low absorption eigenstate on the sphere. Cases I and II are special cases of Case III when either $\beta_{i}$ or $\beta_{r}$ is zero, respectively.

Figures 4, 5, and 6 give the computed evolution of the ellipse of polarization and its parameters (ellipticity and a zimuth) for one example of cases I, II, and III, respectively. In (a) of these figures the trajectories of $\chi\left(z, \chi_{0}\right)$ for different initial states are drawn and in (b), the ellipticity and azimuth for one initial state are plotted as functions of distance $z$ along the direction of propagation. The results conform with the conclusions that we reached above.

\section{Cholesteric Liquid Crystal}

The evolution of the ellipse of polarization when light propagates along the helical axis (the $z$ axis) is given by Eq. (4), starting from the initial state $\chi_{0}$ at $z=0$. As explained in Ref. 1 , the first term in that equation, denoted by $u\left(z, u_{0}\right)$ (where $u_{0}=\chi_{0}$ ), gives the complex-variable description of the polarization ellipse in a space-rotating coordinate system that coincides with the principal axes of the molecular planes of the liquid crystal. Using Eq. (6), $u\left(z, u_{0}\right)$ takes the form of Eq. (7)

$$
u\left(z, u_{0}\right)=\left[A^{\prime}+B^{\prime} \exp (i 2 \beta z)\right] /\left[C^{\prime}+D^{\prime} \exp (i 2 \beta z)\right]
$$

where

$$
\begin{aligned}
& A^{\prime}=g_{0}+(\alpha+\beta) \chi_{0}, \\
& B^{\prime}=-g_{0}-(\alpha-\beta) \chi_{0}, \\
& C^{\prime}=-(\alpha-\beta)+g_{0} \chi_{0}, \\
& D^{\prime}=(\alpha+\beta)-g_{0} \chi_{0} .
\end{aligned}
$$

Because $\alpha$ and $g_{0}$ are real, $\beta$ [given by Eq. (5b)] is also real. Thus $\exp (i 2 \beta z)$ will move on the unit circle, completing one period every time a distance $\pi / \beta$ is traversed. We have a condition similar to that described in Case I of Sec. II. The evolution of $u\left(z, u_{0}\right)$ in the circular complex-plane representation (with the two circular polarizations as basis states) is described by a circle that encloses one of the two orthogonal privileged polarizations $K_{1}$ or $K_{2}$, given by Eq. (28) of Ref. 1. For different initial polarization states $u_{0}\left(\chi_{0}\right)$ we obtain a family of circles enclosing $K_{1}$ and $K_{2}$, orthogonal to the family of circles through these two points (Fig. 7). Because the ellipticity is determined by $\left|u\left(z, u_{0}\right)\right|$, it is easily seen that this quantity is periodic with a period $\pi / \beta$ and goes through one minimum and one maximum within a period when $u\left(z, u_{0}\right)$ crosses the real axis. Although the motion of $\exp (i 2 \beta z)$ on the unit circle is uniform (equal increments of $z$ mean equal distances along the circumference) that of $u\left(z, u_{0}\right)$ is not.

To determine the ellipse of polarization with respect to an $x y z$, space-fixed, coordinate system (where $x$ and $y$ are parallel to the principal axes of birefringence of the molecular plane at $z=0$ and $z$ is the helical axis), $u\left(z, u_{0}\right)$ has to be multiplied by $\exp (i 2 \alpha z)$. The resultant trajectory of $u\left(z, u_{0}\right)$ $\exp (i 2 \alpha z)$ is obtained by the superposition of two motions corresponding to its two terms. One motion 

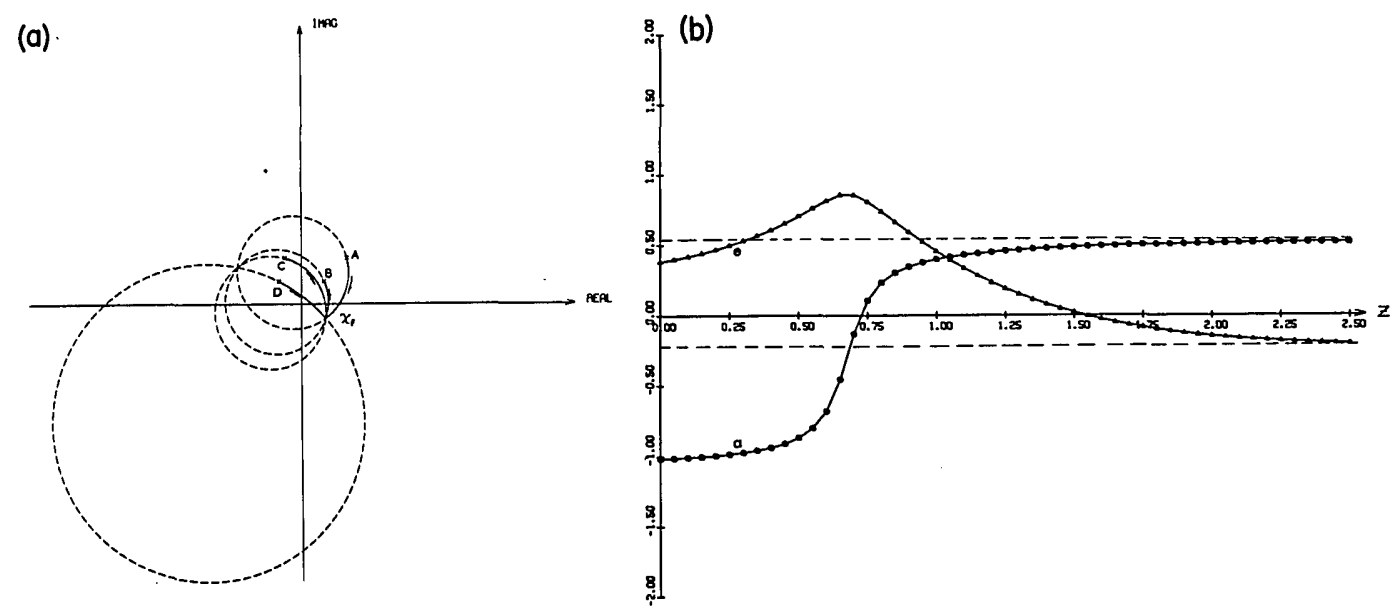

Fig. 5. (a) Same as in Fig. 4(a) for an elliptically dichroic medium whose $N$ matrix has the form of $N_{\text {ed }}$ in Eq. (14) with $p=2, q=1$, and $R=0.866+i .5$. $A, B, C$, and $D$ indicate four different initial states and $\chi_{f}$, the final'state, corresponds to the low-absorption eigenpolarization. (b) Same as in Fig. 4(b) for the trajectory that starts at $C$ in (a). Note the aperiodic nature of the curves and their asymptotic approach to the ellipticity and azimuth of the low-absorption eigenpolarization.
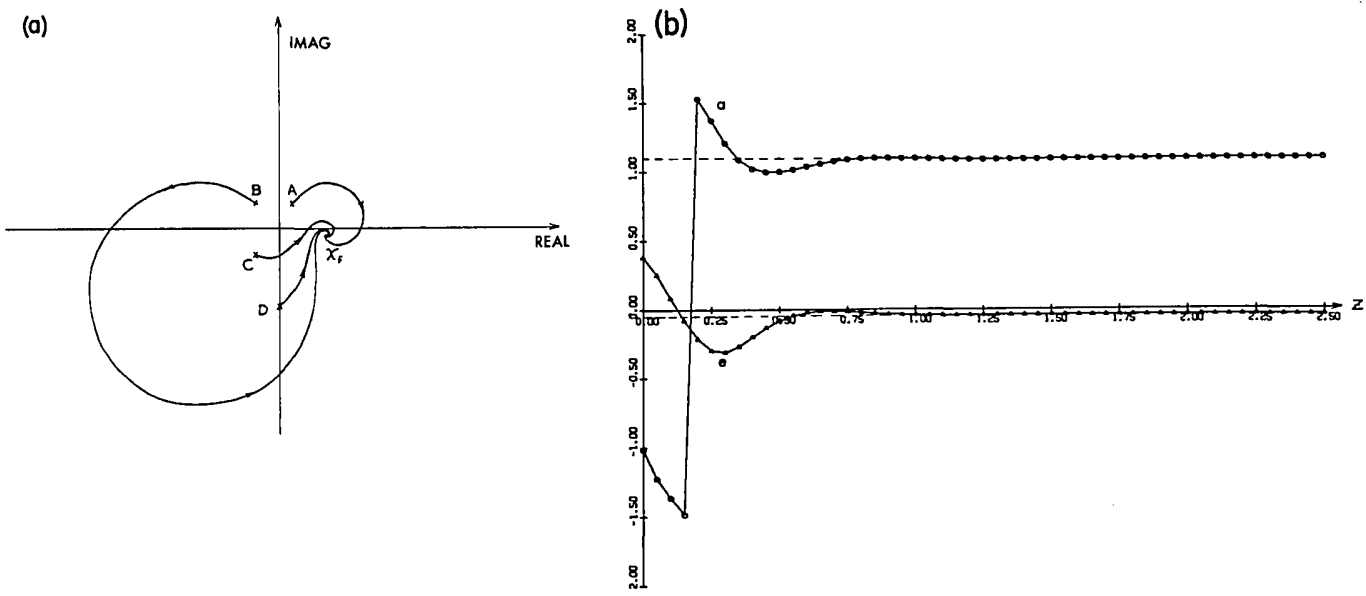

Fig. 6. (a) Same as in Figs. 4(a) and 5(a) for the general homogeneous anisotropic medium that exhibits combined birefringence and dichroism. The $N$ matrix was chosen arbitrarily with elements $n_{11}=1+i 2, n_{12}=2+i 3, n_{21}=3+i 4$, and $n_{22}=4+i 5$. Note the spiraling convergence to the low-absorption eigenpolarization $\chi_{f}$ for the four different initial polarizations $A, B, C$, and $D$. (b) Same as in Figs. 4(b) and $5(\mathrm{~b})$ for the trajectory that starts at $B$ in (a). Note the damped oscillatory convergence to the final ellipticity and azimuth.

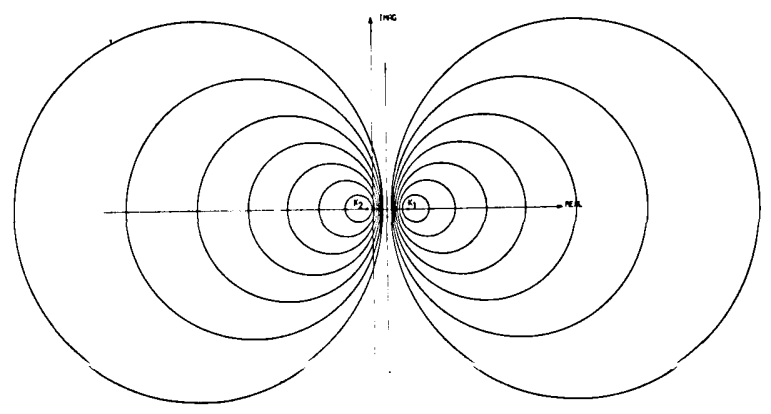

Fig. 7. The trajectories of $u\left(z, u_{0}\right)$ describing the evolution of the polarization ellipse of light propagating along the helical axis of a cholesteric liquid crystal for different initial states, referenced to a space-rotating coordinate system that coincides with the principal axes of the molecular planes. $K_{1}$ and $K_{2}$ are the two privileged polarization ellipses whose axes are forced to remain in alignment with the principal axes of the molecular planes with their ellipticities, unchanged. The complex-plane representation in this figure as well as in (a) of Figs. 8-12 uses the left- and right-circular polarizations as basis states and are represented by the origin and point of infinity, respectively. 
(a)

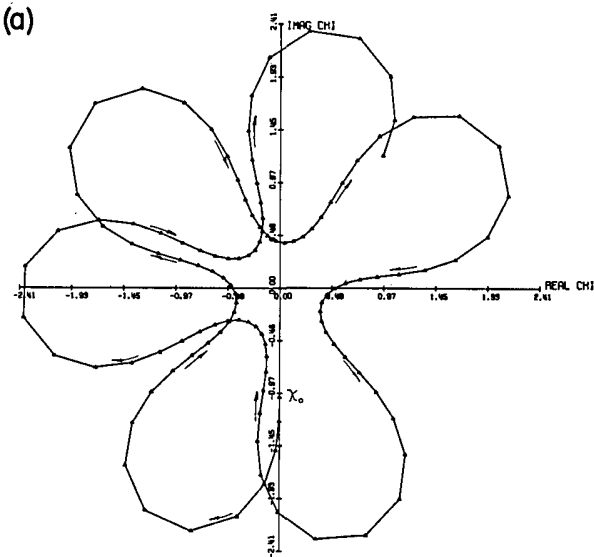

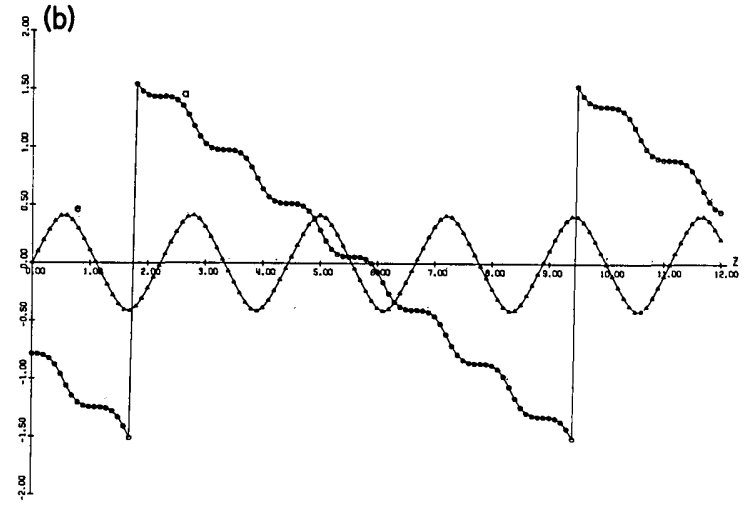

Fig. 8. (a) The evolution of the polarization ellipse along the helical axis of a cholesteric liquid crystal with $\alpha=g_{0}=1$ in a space-fixed coordinate system for an initial state $\chi_{0}=-i$. Two circles can be drawn with center at the origin between which the trajectory of $\chi\left(z, \chi_{0}\right)$ is confined. The nonperiodic nature of the trajectory can be easily seen. (b) The evolution of the ellipticity $e(\Delta)$ and azimuth $a(O)$ along the helical axis for the trajectory of (a). The ellipticity is periodic and the azimuth is not. See text for an explanation of the apparent discontinuities in azimuth both in this figure and in the ones to come.

[due to $u\left(z, u_{0}\right)$ ] is along one of the circles in Fig. 7 (determined by the initial polarization) and the other [due to $\exp (i 2 \alpha z)$ ] is a solid rotation of the circle of $u\left(z, u_{0}\right)$ around the origin at a rate of $2 \alpha$ radians per unit distance $z$ along the helical axis. If the motion around the circle of $u\left(z, u_{0}\right)$ were uniform, the resultant trajectory of the above two motions would be an epicycloid or a hypocycloid. ${ }^{8} \mathrm{Be}-$ cause of the nonuniform rate of motion along the circle [which is determined by the derivative of Eq. (15) with respect to $\exp (i 2 \beta z)$ ] we expect the locus to be a distorted epicycloid or hypocycloid. The trajectory will always be confined between two concentric circles whose center is the origin touching the circle of $u\left(z, u_{0}\right)$. A limiting case results when the locus of $u\left(z, u_{0}\right)$ is the straight line parallel to the imaginary axis bisecting the distance between the two points $K_{1}$ and $K_{2}$, i.e., if the initial state $\chi_{0}$ is such that

$$
\begin{aligned}
\operatorname{Re} X_{0} & =\operatorname{Re} u_{0}=\frac{1}{2}\left(k_{1}+k_{2}\right) \\
& =\alpha / g_{0}
\end{aligned}
$$

In Eq. (17) the values of $K_{1}$ and $K_{2}$ from Eq. (28) of Ref. 1 have been substituted. This limiting case corresponds to a trajectory that passes through the point at infinity and is only limited to the outside of a circle with center at the origin and radius equal to $\alpha / g_{0}$.

Examples of trajectories that describe the evolution of the ellipse of polarization of light along the helical axis of a cholesteric liquid crystal are shown in (a) of Figs. 8-12. These are computer- plotted using Eq. (14). Part (b) of these figures show the evolution of ellipticity and azimuth with distance $z$ along the helical axis. In Figs. 8-10 the parameters $\alpha$ and $g_{0}$ of the liquid crystal are both taken as
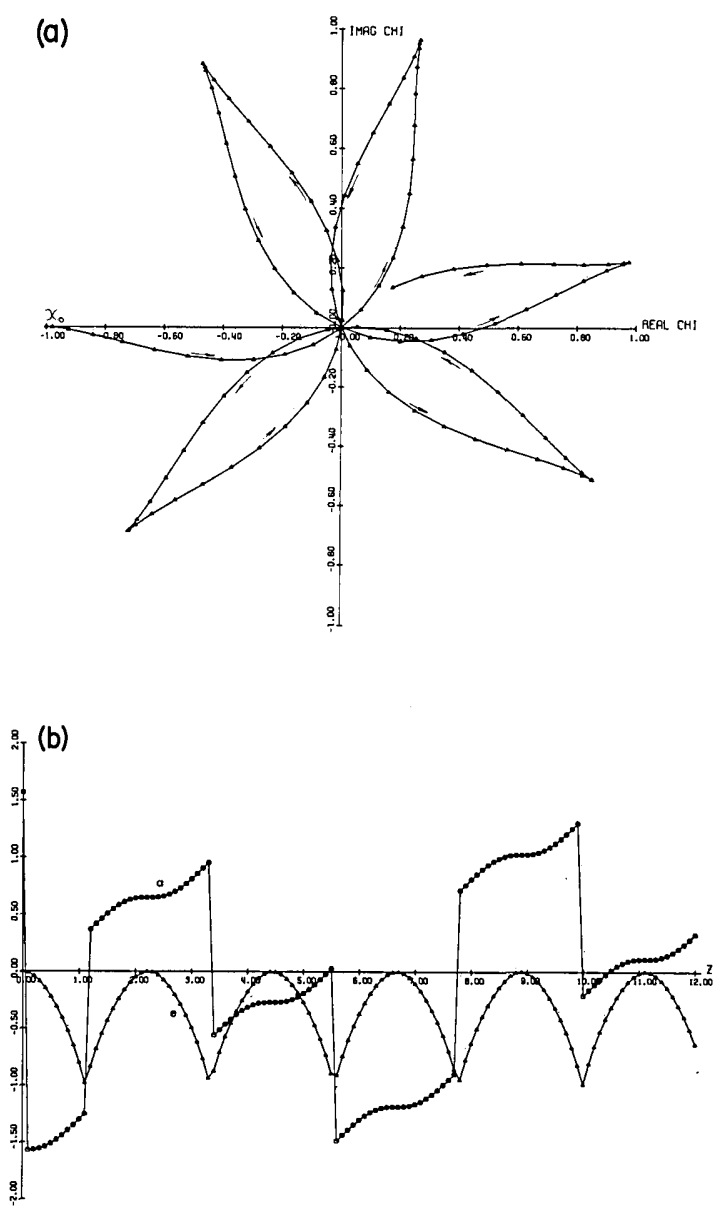

Fig. 9. (a) Same as in Fig. 8(a) for a different initial state $\chi_{0}=$ -1. The trajectory of $\chi\left(z, \chi_{0}\right)$ has overlapping multilobes (leaves). Note that the limiting inner circle has collapsed to a point (the origin). (b) The ellipticity $e(\Delta)$ and azimuth $a(0)$ for the trajectory of $(a)$. 

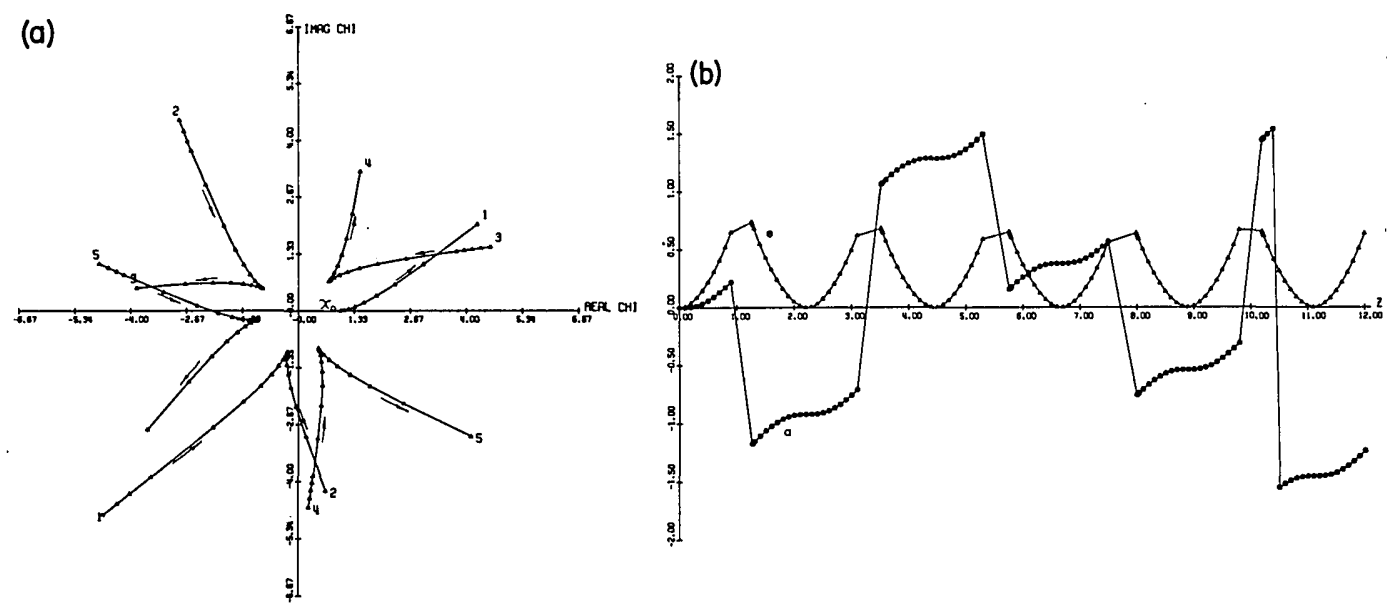

Fig. 10. (a) Same as in Figs. 8(a) and 9(a) for still another initial state, $\chi_{0}=1$. In this case $u\left(z, u_{0}\right)$ becomes the straight line parallel to

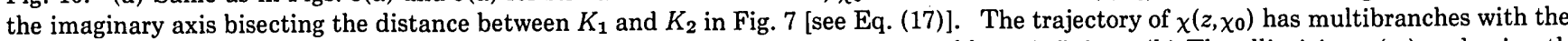
limiting outer circle at infinity. Points marked with the same number join smoothly at infinity. (b) The ellipticity $e(\Delta)$ and azimuth $a$ (O) for the trajectory of (a).

(a)

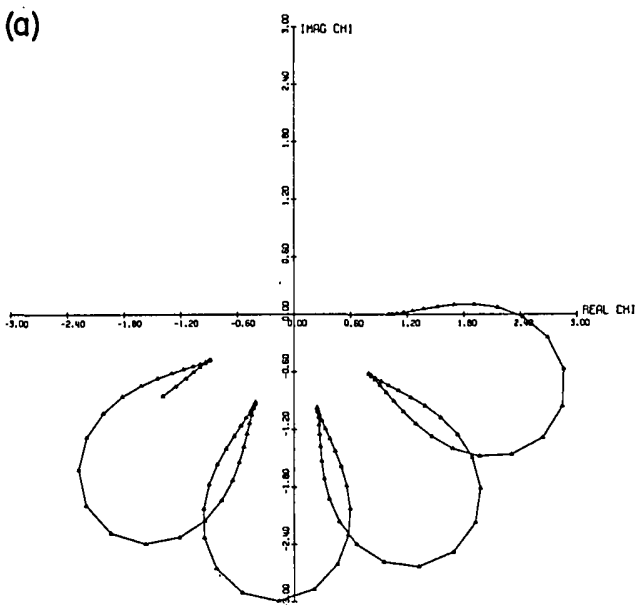

\&i (b)

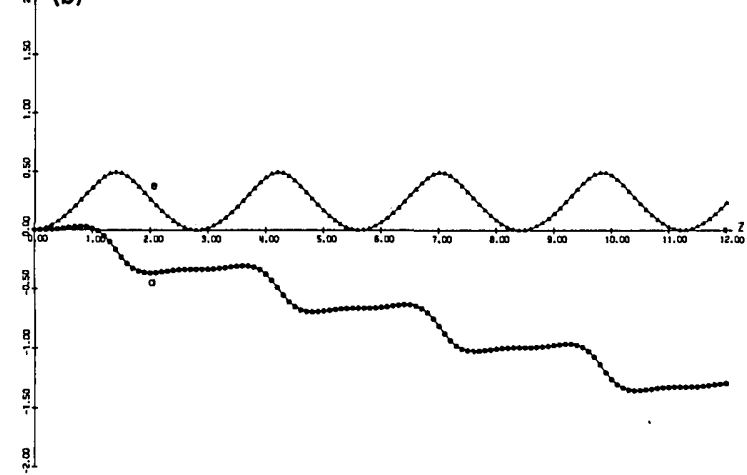

Fig. 11. (a) Same as in Figs. 8(a), 9(a), and 10(a) for a liquid crystal with the same pitch $(\alpha=1)$ but with different molecular birefringence $\left(g_{0}=0.5\right)$. The initial polarization state is $\chi_{0}=1$. This trajectory clearly resembles a hypocycloid, perhaps better than any other figure in this group of trajectories. (b) The ellipticity $e(\Delta)$ and azimuth $a(0)$ for the liquid crystal and trajectory of (a).
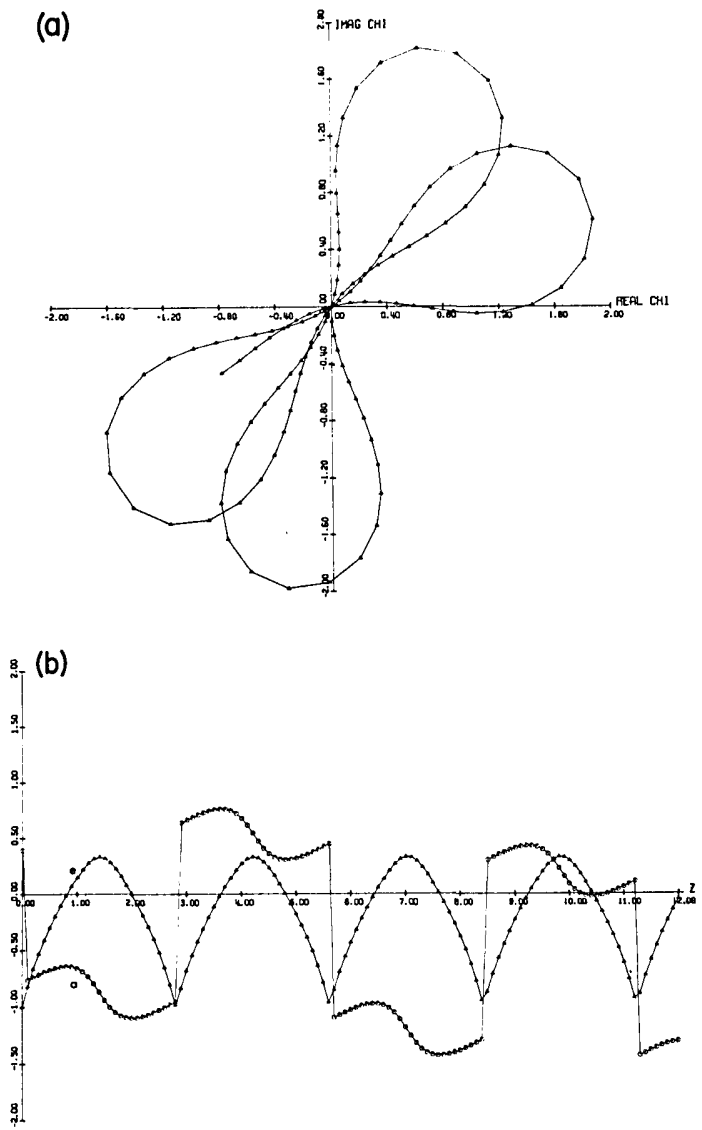

Fig. 12.

(a) Same as in Figs. 8(a), 9(a), and

10(a) except that the crystal now has a different pitch $(\alpha=0.5)$ with $g_{0}$ the same $\left(g_{0}\right.$ $=1$ ). The initial state is $\chi_{0}=0$. (b) The ellipticity $e \quad(\Delta)$ and azimuth $a$ (O) for the liquid crystal and trajectory of (a). 
unity. Figures 8,9 , and 10 are obtained by choosing three different initial polarization states (all of which are linear) $\chi_{0}=-i,-1$, and +1 , respectively. Figure 8(a) represents the general condition of a trajectory confined between two concentric circles with center at the origin. Figures 9(a) and 10(a) correspond to the two limiting cases when the inner circle collapses to a point (the origin) and the outer circle expands to infinity, respectively. Figures $11(a)$ and 12(a) are obtained when the birefringence of the molecular planes $\left(g_{0}\right)$ and the pitch of the helix $(\alpha)$ are changed (one at a time), respectively. All of these trajectories are open, nonrepetitive, and multilobed [Figs. 8(a), 9(a), and 12(a)] or multibranched [Figs. 10(a) and 11(a)]. Figure 11(a), in particular, shows close resemblance to the hypocycloid. Figures 9(a), 10 (a), and 12(a) show exceptions to the rule that was previously stated in ref. 1 that no polarization state other than the privileged polarization $K_{1}$ and $K_{2}$ repeats itself. In. Figs. 9(a), 10(a), and 12(a) the leftor right-circularly polarized state repeats itself every time the trajectory passes through the origin or infinity. This, however, does not imply periodicity, because the polarization passes through the circular state in a different way each time the trajectory passes through the origin or infinity.

From (b) of Figs. 8-12 it is readily observable that the ellipticity of the ellipse of polarization is periodic while the azimuth is not. The azimuth also appears to make several discontinuous jumps. Closer inspection shows that these jumps are of two types and do not represent true discontinuities. The first type of discontinuity occurs when the azimuth reaches a value of $\pi / 2$ or $-\pi / 2$ at which a jump of $\pm \pi$ takes place. This readily follows from the fact that the range of azimuth values is limited between $-\pi / 2$ and $+\pi / 2$ and that these limiting azimuths are physically indistinguishable. The second type of discontinuity occurs when the ellipse of polarization goes through the left or right circular states, which are of indeterminate azimuth. This results when $\chi\left(z, \chi_{0}\right)$ passes through zero [Figs. 9(a) and 12(a)] or infinity [Fig. 10(a)], respectively. Because the azimuth is determined by half the angle of $\chi$ [Eq. (10), Ref. 1], a jump of $\pi / 2$ is expected when $\chi\left(z, \chi_{0}\right)$ passes through the origin or the point at infinity.

\section{Summary}

The nature of the analytic solutions that we have obtained recently ${ }^{1}$ for the evolution of the ellipse of polarization of light in homogeneous anisotropic media and cholesteric liquid crystals has been examined. When the differential propagation matrix $N$ of a homogeneous anisotropic medium satisfies Eq. (13), the point that represents the state of polarization in the complex plane or on the Poincare sphere moves along a circle enclosing one eigenpolarization (pure birefringence) or along an arc of a circle ending at the low-absorption eigenpolarization (pure dichroism). The general medium exhibiting combined birefringence and dichroism leads to spiral convergence to the low-absorption eigenpolarization.

The solution for light propagation along the helical axis of a cholesteric liquid crystal yields a variety of shapes for the trajectory describing the evolution of the ellipse of polarization with distance $z$, starting from an initial state $\chi_{0}$ at $z=0$. These have been proved to be distorted hypo- or epicycloids and appear in the form of open nonrepetitive multilobed or multibranched trajectories depending on the initial state $\chi_{0}$ and the properties of the liquid crystal.

This work was supported by the National Science Foundation.

\section{References}

1. R. M. A. Azzam and N. M. Bashara, J. Opt. Soc. Am. 62, 1252 (1972).

2. R. C. Jones, J. Opt. Soc. Am. 38, 671 (1948).

3. The cholesteric liquid crystal problem has also been treated by C. W. Oseen, Trans. Faraday Soc. 29, 883 (1933); Hl. De Vries, Acta Crystallogr. 4, 219 (1951); G. H. Conners, J. Opt. Soc. Am. 58, 875 (1968); D. W. Berreman and T. J. Scheffer, Mol. Crystallogr. Liquid Crystallogr. 11, 395 (1970), J. Opt. Soc. Am. 62, 502 (1972); A. S. Marathay, J. Opt. Soc. Am. 61, 1363 (1971), Optics Commun. 3, 369 (1971). This is not an exhaustive list.

4. The proof follows from finding the ratios $A / C$ and $B / D$ using Eq. (8) and noticing that the condition of their independence of $\chi_{0}$ leads to the definition of $\beta$ in Eq. (3). That these ratios give the eigenpolarizations can be checked by substitution in Eq. (7).

5. R. M. A. Azzam and N. M. Bashara, J. Opt. Soc. Am. 62, 222 (1972).

6. R. M. A. Azzam and N. M. Bashara, Optics Commun. 5, 319 (1972).

7. A conformal transformation preserves the shape of any curve in the immediate vicinity of a point in the complex plane. The spiraling behavior of $\exp (i 2 \beta z)$ around the origin (or the point at infinity) is therefore reproduced by $\chi\left(z, \chi_{0}\right)$ around the low-absorption eigenpolarization.

8. P. K. Rees, Analytic Geometry (Prentice-Hall, Englewood Cliffs, N. J., 1970), p. 177. 